\title{
Acousto-Optic Devices Based on Potassium Rare-Earth Tungstates Laser Crystals
}

\author{
D.Yu. VelikovskiI ${ }^{a, *}$, V.E. PozhaR ${ }^{a}$ AND M.M. MAzUR ${ }^{b}$ \\ ${ }^{a}$ Scientific and Technological Center of Unique Instrumentation, Russian Academy of Sciences (STC UI RAS), \\ Butlerova str. 15, 117342, Moscow, Russia \\ ${ }^{b}$ Federal Research Institute for Physical Technical and Radioengineering Measurements (VNIIFTRI), \\ 141570 Moscow region, Mendeleyevo, Russia
}

\begin{abstract}
We present a family of original acousto-optical devices. They are based on new acousto-optical materials $\mathrm{K} R E\left(\mathrm{WO}_{4}\right)_{2}$, where $R E=\mathrm{Y}, \mathrm{Gd}, \mathrm{Yb}, \mathrm{Lu}$. A combination of features such as radiation resistance, optical biaxiality and appreciable acousto-optical figure of merit, makes possible creation of polarization-insensitive modulator with almost $100 \%$ of diffraction efficiency. The deflector with wide angular aperture and broad range of deflection, and collinear tunable filter are also suggested.
\end{abstract}

DOI: 10.12693 /APhysPolA.127.75

PACS: $42.70 . \mathrm{Hj}, 42.79 . \mathrm{Jq}, 42.60 . \mathrm{Fc}, 43.35 . \mathrm{Sx}$

\section{Introduction}

With the progress of laser technologies, new crystalline materials are synthesized and utilized. The crystal $\mathrm{KGd}\left(\mathrm{WO}_{4}\right)_{2}$ (short: KGW) [1] is one of the most famous due to a very high threshold of laser damage: up to $170 \mathrm{GW} / \mathrm{cm}^{2}$ for $\tau=20 \mathrm{~ms}$ pulses [2]. It is widely used in laser technics as lasing material [3]. Moreover, it has rather good acousto-optical (AO) properties [4], as well as the group of double potassium rare-earth tungstates $\mathrm{K} R E\left(\mathrm{WO}_{4}\right)_{2}$, where $R E=\mathrm{Yb}, \mathrm{Lu}$, and also $\mathrm{Y}[5,6]$. It has been demonstrated for the first time [5] that their AO figure of merit $M_{2}$ is comparable with that of $\mathrm{LiNbO}_{3}$ and better than $M_{2}$ of $\mathrm{SiO}_{2}$, so they can efficiently replace quartz in various laser applications. Moreover, these monoclinic crystals (symmetry class $2 / m$ ) are optically biaxial, transparent in visible and infrared ranges (0.4-5.5 $\mu \mathrm{m})$, and demonstrate significant anisotropy.

These facts open new potentials in development of optical functional devices. First of all, basic AO devices (modulators, deflectors, tunable filters) made of those materials are able to withstand high power laser radiation, which is very important for laser industrial applications. For example, acousto-optic (AO) mode lockers for high power lasers are usually made of $\alpha-\mathrm{SiO}_{2}$, so they require a cooling system due to high acoustic power supplied. The laser crystals $\mathrm{K} R E\left(\mathrm{WO}_{4}\right)_{2}$ (hereafter $\mathrm{K} R E \mathrm{~W}$ ) do not need cooling as they have a higher acousto-optical efficiency. Secondly, some kind of hybrid devices can be designed, which combine laser and acousto-optic properties and provide light generation and light operation on the same crystal element.

In this paper we present three AO devices based

*corresponding author; e-mail: dgvelikovsky@gmail.com on the KREW crystals: polarization-insensitive modulator, wide-angle deflector, and collinear tunable filter. The first device is based on isotropic (polarizationmaintaining) diffraction, while the others - on anisotropic one.

\section{Polarization-insensitive modulator}

New AO modulator made of KGW single crystal was developed, created and tested (Fig. 1). It uses the quasilongitudinal ultrasonic wave (QL) propagating along the axis $N_{g}$ of dielectric (optical) coordinate system (Fig. 2). The principle of operation is following.

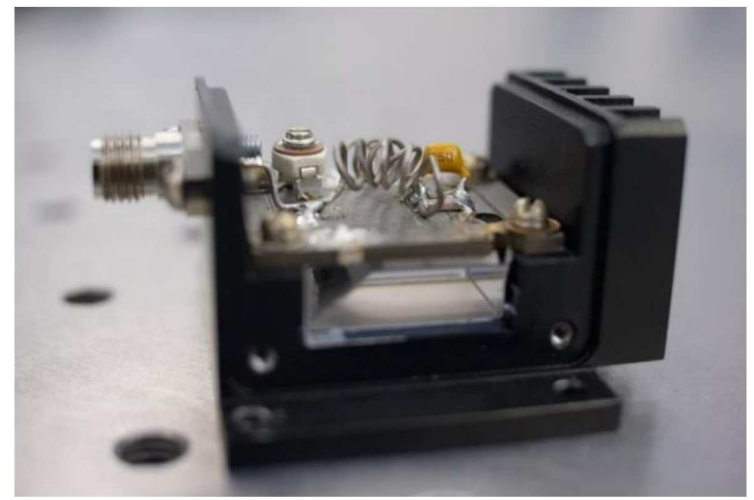

Fig. 1. Acousto-optical modulator made on KGW single crystal.

At the acoustic wave, both ordinary and extraordinary optical waves can diffract simultaneously, however at different incident angles. In the case of isotropic diffraction, the Bragg angles depend on refractive indices of the waves in the following manner:

$$
\Theta_{\mathrm{B}}^{m}=\frac{K}{2 k n_{m}}, \quad \Theta_{\mathrm{B}}^{g}=\frac{K}{2 k n_{g}},
$$




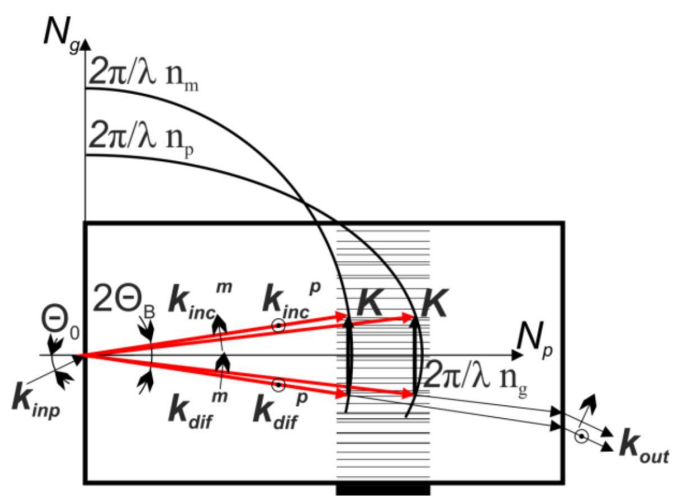

Fig. 2. Wave vector diagram for polarizationindependent modulator.

where $k$ and $K$ are the wave numbers of light and ultrasonic waves. Therefore, the both components of naturally polarized light, which are incident on the input face at the angle $\Theta_{0}=n_{m} \Theta_{\mathrm{B}}^{m}=n_{g} \Theta_{\mathrm{B}}^{g}$, propagate after refraction inside the crystal at the Bragg angles, so they can be diffracted simultaneously. After diffraction and refraction at the back face of the crystal these components propagate in parallel directions.

Thus, the modulator can operate two polarization components of light at the same time and can be regarded as a bi-polarization device. Fortunately, the diffraction efficiency values for both polarizations are of the same order (the ratio of AO figure of merit $M_{2}$ is 6:10 for $\mathrm{KGW}$ and 10:11 for $\mathrm{KLuW}$ ), hence, for any polarization of the incident light, the polarization direction remains the same after diffraction and, therefore, the modulator in fact is a polarization-independent device.

The diffraction coefficient of the created modulator reaches $80 \%$ at $532 \mathrm{~nm}$ with $1 \mathrm{~W}$ of driving power at the frequency about $85 \mathrm{MHz}$ for the transducer width of $2 \mathrm{~mm}$ and the length $22 \mathrm{~mm}$. The diffraction coefficient reaches as much as $98 \%$ at $633 \mathrm{~nm}$ with power $2.5 \mathrm{~W}$. At near infrared the efficiency is lower: $66 \%$ with power $4 \mathrm{~W}$ at $1.06 \mu \mathrm{m}$. Therefore, the diffraction efficiency of this KGW modulator is only three times lower than the efficiency of $\mathrm{AO}$ modulator usually made of $\mathrm{TeO}_{2}$. They use ultrasound $L$-mode propagating along [001] direction and light polarization [100] with $M_{2}=34.5 \times 10^{-15} \mathrm{~s}^{3} / \mathrm{kg}$ [7].

An important feature of the developed modulator is a capability to withstand the high-power laser radiation. AO cell made on KGW crystal maintains its initial temperature in experiment being exposed to $20 \mathrm{~kW} / \mathrm{cm}^{2}$ continuous laser emissions indicating the higher level of radiation resistance than that of paratellurite crystal, which gets $20^{\circ} \mathrm{C}$ warmer (in the same conditions).

\section{Wide-angle deflector}

The fact that potassium tungstates crystals $\mathrm{K} R E \mathrm{~W}$ are biaxial materials makes possible the development of new kinds of $\mathrm{AO}$ units on their base, which cannot be made with use of uniaxial crystals usually utilized in acousto-optics. For example, the wide-angle deflector with wide angular aperture and great tuning range can be produced [8]. For this purpose, one must use the geometry with ultrasound wave vector being a tangent to wave surface of two modes so that non-critical phase matching condition is satisfied (Fig. 3). In this case beams with wide angular spectrum $\left(\Delta \Theta_{1}\right)$ can be diffracted efficiently while the deflection angle is proportional to acoustic frequency variation $\left(\Delta \Theta_{2} \sim \Delta K \sim \Delta f\right)$. Such AO deflector can operate angular-extended beams, in particular, profiled laser beams, focused radiation, etc. It is useful for imaging applications. And also, the switching time of a high-speed AO modulator is approximately equal to the acoustic transit time through the waist of the focused beam $\tau=d / V_{\text {ac }} \sim 1 / \Delta \Theta_{1}$, estimated as $20 \mathrm{~ns}$ and less.

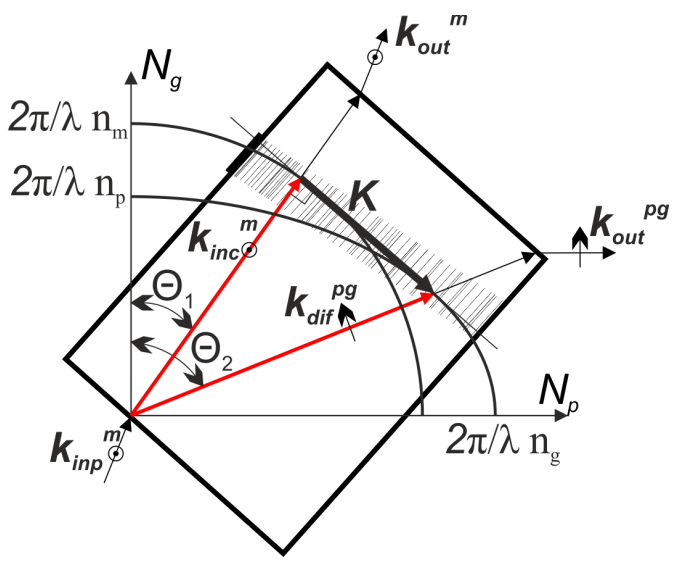

Fig. 3. Wave vector diagram for wide-angle deflector.

In such a way, this deflector combines amplitude and angular effects that make new methods of the laser beam control possible. Unfortunately, this geometry of AO interaction requires quite high driving ultrasonic frequency

$$
f=\frac{V_{s}}{\lambda} \frac{\sqrt{\left(n_{g}^{2}-n_{m}^{2}\right)\left(n_{m}^{2}-n_{p}^{2}\right)}}{n_{m}}
$$

up to a few gigahertz for visible range. The minimum velocity of shear ultrasonic waves among discussed crystals is provided by KLuW, which also demonstrates the highest acoustic anisotropy. For this crystal the driving frequencies at wavelengths $1.064 \mu \mathrm{m}$ and $1.55 \mu \mathrm{m}$ are 1.1 and $0.8 \mathrm{GHz}$, respectively. These rather high values can be achieved in modern $\mathrm{AO}$ units. In the middle infrared range the frequency is lower.

\section{Collinear filter}

Collinear AO interaction is always of particular attention as it provides the highest spectral resolution and the minimum driving power. For spectroscopic and imaging applications the wide angular aperture is necessary, that is why one needs to choose directions parallel to the dielectric axes $N_{m}, N_{p}$, and $N_{g}$. The most suitable acoustic 
frequencies correspond to $N_{g}$ axis. The AOTF of this geometry (Fig. 4) is characterized with an acoustic column walk-off like the collinear AOTF on $\alpha-\mathrm{SiO}_{2}[9]$. Among the four discussed materials (Table), KYbW crystals can provide the best spectral resolution $\delta \lambda \sim 1 / \Delta n$ because of the highest refractive index difference $\Delta n=n_{p}-n_{m}$. The bandwidth for the interaction length of $1 \mathrm{~cm}$ can be estimated as $\delta \lambda=0.7 \mathrm{~nm}$. Unfortunately, the driving power cannot be calculated, because only a part of photoelastic matrix elements has been measured till now.

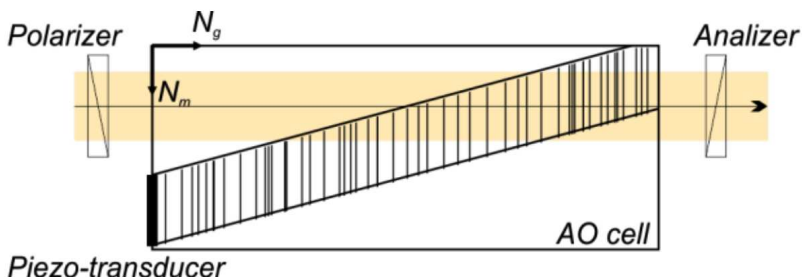

Fig. 4. Schematic diagram of collinear filter on $\mathrm{KRE}\left(\mathrm{WO}_{4}\right)_{2}$ single crystal.

TABLE

Collinear AOTF driving frequency and bandwidth at $\lambda=633 \mathrm{~nm}$ for four $\mathrm{KRE}\left(\mathrm{WO}_{4}\right)_{2}$ materials.

\begin{tabular}{l|c|c}
\hline \hline & $f[\mathrm{MHz}]$ & $\partial \lambda[\mathrm{nm}]$ \\
\hline $\mathrm{KY}\left(\mathrm{WO}_{4}\right)_{2}$ & 200 & 0.76 \\
$\mathrm{KGd}\left(\mathrm{WO}_{4}\right)_{2}$ & 150 & 1.05 \\
$\mathrm{KYb}\left(\mathrm{WO}_{4}\right)_{2}$ & 190 & 0.74 \\
$\mathrm{KLu}\left(\mathrm{WO}_{4}\right)_{2}$ & 170 & 0.83
\end{tabular}

\section{Conclusion}

The new group of acousto-optic materials, which are potassium rare-earth tungstates, possess an original complex of features: resistance to high-power laser radiation, optical biaxiality and high AO figure of merit. Therefore, a new family of $\mathrm{AO}$ units and devices can be created: modulators, deflectors, filters. They can provide both quantitative and qualitative advantages. New AO modulator on $\mathrm{KGd}\left(\mathrm{WO}_{4}\right)_{2}$ single crystal for non-polarized high-power light radiation was developed and tested. It demonstrated almost $100 \%$ of diffraction coefficient. Extra capabilities may probably be exhibited with implementation of anisotropic diffraction. However, the appropriate elasto-optical coefficients must be experimentally determined.

A very promising $\mathrm{AO}$ element developed is the deflector with wide angular aperture and broad range of deflection, which can control high-power Gaussian-profiled laser beams. It is capable to operate with more intensive laser beams than the existing $\mathrm{TeO}_{2}$ modulators, requiring somewhat higher driving power, but providing an efficient modulation of arbitrary-polarized radiation.

\section{Acknowledgments}

The present work was supported by the RFBR grants 13-02-01254, 13-02-12210, and 13-00-14379.

\section{References}

[1] A.A. Pavlyuk, L.I. Yudanova, O.G. Potapova, Inorg. Mater. 33, 64 (1997).

[2] I.V. Mochalov, Opt. Eng. 36, 1660 (1997).

[3] A.A. Kaminskii, J.B. Gruber, S.N. Bagaev, K. Ueda, U. Hommerich, J.T. Seo, D. Temple, B. Zandi, A.A. Kornienko, E.B. Dunina, A.A. Pavlyuk, R.M. Klevtsova, F.A. Kuznetsov, Phys. Rev. B 65, 125108 (2002).

[4] M.M. Mazur, D.Yu. Velikovskii, F.A. Kuznetsov, L.I. Mazur, A.A. Pavlyuk, V.E. Pozhar, V.I. Pustovoit, Acoust. Phys. 58, 658 (2012).

[5] M.M. Mazur, F.A. Kuznetsov, L.I. Mazur, A.A. Pavlyuk, V.I. Pustovoit, Inorg. Mater. 48, 67 (2012).

[6] M.M. Mazur, D.Yu. Velikovskiy, L.I. Mazur, A.A. Pavluk, V.E. Pozhar, V.I. Pustovoit, Ultrasonics 54, 1311 (2014).

[7] M. Bass, C. DeCusatis, J. Enoch, V. Lakshminarayanan, G.-F. Li, C. MacDonald, V. Mahajan, E. Van Stryland, Handbook of Optics, vol. 2, McGraw-Hill, New York 1995.

[8] Hyuk Lee, IEEE Trans. Ultrason. Ferroelectr. Freq. Control UFFC-34, 485 (1987).

[9] I.C. Chang, Appl. Phys. Lett. 25, 323 (1974). 